\title{
Simulation of the Electromechanical Activity of the Heart Using XMR Interventional Imaging
}

\author{
Maxime Sermesant ${ }^{1}$, Kawal Rhode ${ }^{1}$, Angela Anjorin ${ }^{1}$, Sanjeet Hegde ${ }^{1}$, \\ Gerardo Sanchez-Ortiz ${ }^{2}$, Daniel Rueckert ${ }^{2}$, Pier Lambiase ${ }^{3}$, Clifford Bucknall ${ }^{3}$, \\ Derek Hill ${ }^{1}$, and Reza Razavi ${ }^{1}$ \\ 1 Division of Imaging Sciences, Guy's, King's \& St Thomas' School of Medicine, \\ King's College London, 5th Floor Thomas Guy House, Guy's Hospital, London, UK \\ 2 Department of Computing, Imperial College London \\ 3 Department of Cardiology, St Thomas' Hospital, London
}

\begin{abstract}
Simulating cardiac electromechanical activity is of great interest for a better understanding of pathologies and therapy planning. Design and validation of such models is difficult due to the lack of clinical data. XMR systems are a new type of interventional facility in which patients can be rapidly transferred between x-ray and MR systems. Our goal is to design and validate an electromechanical model of the myocardium, using this XMR system. The proposed model is computationally fast and uses clinically observable parameters. We present the integration of anatomy, electrophysiology, and motion from patients. Pathologies are introduced in the model and the simulations are compared to measured data. Initial qualitative comparison is encouraging. Quantitative local validation is in progress. Once validated, these models will make it possible to simulate different interventional strategies.
\end{abstract}

\section{Introduction}

Simulation of normal and pathological electromechanical activity in the heart is of great current interest [6]. The validation of such models is extremely difficult, especially in the case of human pathology. In this paper we describe an electromechanical model of the heart that is designed to simulate the behaviour of the heart during normal and abnormal heart rhythms. The long term aim of this work is to devise a technique to make electrophysiology studies (EPS) to correct abnormal heart rhythms less invasive and more successful. We use a catherisation lab incorporating both MRI and conventional fluoroscopy (XMR) to enable us to collect data from patients about cardiac anatomy, motion and electrical activity during their catherisation. This data is integrated into a common coordinate system using device tracking and registration. A sub-set of this data can be used to initialise the model, and simulate the observed pathology. The modelled behaviour can then be validated using the rest of the collected data. This approach is applied to two patients, one with a ventricular tachycardia, and the second with left bundle branch block. 
Modelling the Cardiac Electromechanical Activity. Combination of medical image analysis and organ simulation opens new perspectives in clinical applications. The integration of knowledge from biology, physics and computer science makes it possible to combine in vivo observations, in vitro experiments and in silico simulations. During recent decades, there have been great advances in the knowledge of heart function, from the nanoscopic to the mesoscopic scale. A global integrative model of this organ becomes conceivable.

Understanding and modelling cardiac electrophysiology, studying the inverse problem from body surface potentials and direct measurement of heart potentials are active research areas. Moreover, many constitutive laws have been proposed in the literature for the mechanical modelling of the myocardium [12].

All these models need rich electromechanical data for validation. Macroscopic measures of the electrical and mechanical activities on the same heart are now available [7]. However, these measures are difficult to obtain and very invasive. Only animal data is available this way. None of these models have been validated against in vivo human data.

The presented work makes possible the validation of an electromechanical model of the human myocardium, due to integration of electrical and mechanical clinical data. The modelling choices are guided by both the nature of the measures (e.g. only the extracellular potential is available for electrophysiology) and their resolution (e.g. spatial and temporal resolution of MR). This model can help to make the most of these measurements, as well as allow simulation of different pathologies and interventional strategies.

Electrophysiological Pathologies and Interventions. Cardiac arrhythmias are the cause of considerable morbidity and even occasional mortality. Tachyarrhythmias (fast heart rhythms) can originate from ectopic foci of electrical depolarisation or from abnormal conduction pathways in the myocardium. The treatment of choice for patients with tachyarrhythmias is radio-frequency ablation (RFA), where the abnormal electrical focus or pathway is ablated by applying radio-frequency energy. For patients with heart failure associated with ventricular asynchrony, the treatment of choice is biventricular pacing through a pacing device.

An electrophysiology study (EPS) is performed prior to these interventions: an electrical measurement catheter is inserted into the appropriate chamber of the heart and the electrical activity on the endocardial surface is measured.

Two Cardiac Electrophysiology Mapping Systems Used. The contact mapping system employs the Constellation catheter from Boston Scientific, a multi-electrode basket catheter. The basket adapts to the endocardial surface and deforms so that contact is maintained through the cardiac cycle.

The non-contact mapping system Ensite from Endocardial Solutions employs a catheter containing a flexible balloon made from a wire mesh. The balloon floats inside the desired cardiac chamber and does not contact the endocardial surface. A second catheter (roving catheter) is inserted and emits a radiofrequency signal from its tip that is detected by the balloon. It is used to map 
the endocardium. The electrical activity measured by the balloon is then extrapolated to this surface.

XMR Interventional Imaging. The XMR interventional suite at King's College London (Guy's Hospital Campus) comprises a 1.5 T MR scanner (Philips Intera I/T) and a mobile cardiac x-ray set (Philips BV Pulsera). The patient can be easily moved between the two systems using a specially modified sliding MR table top that docks with and transfers patients to a specially modified x-ray table. The docking and transfer takes less than 60 seconds.

Although it is possible to acquire MR images and x-ray images of the same patient during a procedure, the XMR system has no inherent ability to register these images. We have previously described the validation of a novel XMR registration technique that is applicable to the sliding table XMR configuration [9].

\section{Clinical Cases}

Clinical Case 1: Contact Mapping System. Patient 1, male aged 15, had an intermittent ventricular tachycardia that was to be treated by EPS and RFA. Initially, MR imaging was performed, using SSFP three-dimensional multiphase sequence and myocardial motion imaging was performed in both short axis (SA) and long axis (LA) views using SPAMM tagged imaging. The patient was then transferred to the x-ray system. Dynamic biplane tracked x-ray images were acquired with the catheters in place and at the same time the electrical activity was recorded from the Constellation catheter during two ventricular ectopic beats. The patient then underwent a successful RFA.

Clinical Case 2: Non-Contact Mapping System. Patient 2, male, aged 68 , had poor left ventricular function following a myocardial infarction. The patient was to undergo EPS and programmed pacing to assess the optimal location of pacing wires for biventricular pacing. Initially, MR imaging was performed using SSFP 3D multiphase sequence and myocardial motion imaging was performed in both SA and LA views using CSPAMM spiral tagged imaging sequence. The patient was then transferred to the x-ray system and the myocardial electrical activity was measured. Dynamic biplane tracked x-ray images were acquired with the catheters in place. The patient then underwent programmed pacing and had a successful pace maker implantation at a later date.

\section{Integration of the Electromechanical Data}

For each of these cases, we have access to different types of information: anatomy from MR 3D volume images, motion with multislice tagged MR images, invasive electrophysiology with the mapping system, position of the mapping system with $\mathrm{x}$-ray images and XMR registration technology.

MR images were segmented to isolate the anatomy of interest using the Analyze software package (Mayo Clinic, Minnesota, USA). And the myocardial motion was extracted by analysis of the tagged MR images with a non-rigid registration approach [4]. 
The combination of these data provide a very rich material for modelling the myocardium. Before using these data for a model, we have to integrate all the different information in the same coordinate system (fig. 31).

\subsection{Mapping Electromechanical Data to Patient Anatomy}

The XMR registration technique aligns the MRI and electrophysiology data to an accuracy of 4-10 $\mathrm{mm}$. There are residual errors due primarily to respiratory motion and errors in the surface location from the EP system (fig. 44).

Clinical Case 1: Rigid Registration. We correct for residual misregistration with a surface to image registration technique based on the Iterative Closest Point algorithm [8]. For each vertex of the basket mesh, we computed the corresponding boundary voxel in the MR image by looking along the normal for a boundary point, defined from gradient value and direction. Then from all the matched vertex/boundary point pairs we estimated the best rigid body transformation, and iterate until convergence.

Clinical Case 2: Non-Rigid Registration. The EnSite system produces a surface representation of the left ventricle. This surface is estimated with the roving catheter, considering the furthest position as end-diastolic. When registered to the segmented MR anatomy with the XMR registration technology, it appears that its shape do not exactly match the left ventricular MR anatomy.

We correct this using a deformable surface, which evolves like a snake method under the influence of an external energy computed from the gradient of the image and adjusting the surface to the image boundaries and an internal energy keeping the surface regular from [5]. For each vertex of the surface, a force is applied on the vertex, proportional to the distance to the closest boundary point.

These transformations make it possible to have the anatomy, motion an electrophysiology information in the same coordinate space (see fig. 33).

\section{Modelling the Cardiac Electromechanical Activity}

Simulation of the Cardiac Electrophysiology. Simulation of the cardiac electrophysiology is usually done using either the Luo-Rudy type of models or the FitzHugh-Nagumo type. The former is based on the simulation of all the different ions present in a cardiac cell along with the different channels. The latter is based on a more global scale, only modelling the potential difference between the intracellular and the extracellular space (the action potential).

As we are more interested in the timing of the wave propagation than in what is happening at the ion scale, and we intend to interpret it in terms of local conductivity, we use a FitzHugh-Nagumo type of model. Moreover, Luo$R u d y$ variables are not observable in vivo, and much too numerous to be adjusted from such data. We use the adapted version for cardiac cells of the FitzHughNagumo equations proposed by [1], in a simplified form:

$$
\begin{aligned}
& \partial_{t} u=\operatorname{div}(D \nabla u)+k u(1-u)(u-a)-u z \\
& \left.\partial_{t} z=-\varepsilon(k u(u-a-1)+z)\right)
\end{aligned}
$$


$u$ : normalised action potential, $z$ : repolarisation variable, $k$, $\varepsilon$ : repolarisation parameters, a: reaction parameter. We can solve these equations on a volumetric tetrahedral mesh (3D Finite Element Method) if we want to compute a whole myocardium propagation or only on triangulated surfaces (2D Finite Element Method), if we want to simulate the propagation on the endocardium. We can also introduce the muscle fibre directions in the computation, through the diffusion tensor $D$, as they intervene in the propagation speed. More details about the electrical model can be found in [11].

Simulation of the Myocardium Contraction. The action potential controls the mechanical contraction of the myocardium. To simulate this phenomenon, the constitutive law for the myocardium must include an active element, responding to an action potential by developing a contraction stress. We use a model based on the one presented in [10], derived from a multi-scale modelling of the myocardium detailed in 3. This model is a combination of a (transverse anisotropic, piecewise) linear visco-elastic passive constitutive law, with an active element creating a contraction stress tensor controlled by the action potential.

$$
M \frac{d^{2} U}{d t^{2}}+C \frac{d U}{d t}+K U=F_{e}+F_{c}
$$

where $M$ is the mass matrix, $C$ the damping matrix, $K$ the stiffness matrix, $U$ the displacement vector, $F_{e}$ the external forces vector and $F_{c}$ the contraction vector. This model makes it possible to simulate the cardiac cycle, by using different boundary conditions depending on the current phase (filling, isovolumetric contraction, ejection, isovolumetric relaxation). The transition from one phase to another is automatically controlled by the pressure and the flow in the simulated model.

\section{Simulation Results}

The model described in section 4 has been applied to simulate the electrical activity and motion in the two clinical cases, and compare this simulated behaviour to the measured electrical and mechanical behaviour.

Clinical Case 1: Simulation of an Ectopic Focus. An ectopic focus can be simulated by introducing an additional depolarising area in the model, located from the measured position with the Constellation catheter, and starting at the time given by the electrical recordings (fig. 5).

The electromechanical model of the myocardium has been registered with the anatomical MR to obtain the patient geometry. Then, the vertices corresponding to the ectopic focus were determined, looking for the closest vertices to the Constellation electrodes where this focus was observed.

The timing of the ectopic beat was deduced from the observation of the electrical recordings, which also comprise several ECG derivations. Then an ectopic beat was simulated, the ectopic excitation taking place during the $\mathrm{P}$ wave, before the normal QRS complex. 


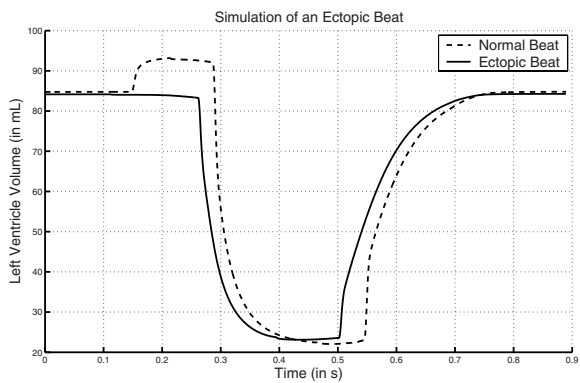

Fig. 1. Consequences on cardiac function parameters: comparison between the simulated normal (dashed) and the simulated ectopic (solid) left ventricle volume.

We can observe in the simulation that the early contraction of the myocardium prevents the atrial contraction from filling the ventricles completely. The ejected volume drops from $71 \mathrm{~mL}$ to $61 \mathrm{~mL}$.

Moreover, the simulation of several ectopic beats leads to an even greater difference, with a smaller end diastolic volume for the ectopic heart. Thus the consequences of arrhythmia on simulated cardiac function are well represented.

Clinical Case 2: Simulation of a Scar with a Left Branch Block. This patient has a scar which can be clearly observed in the late enhancement MR image acquired. This scar led to a left branch block, which is observable in the electrophysiological measures: the excitation in the left ventricle only comes from the septum area, without any other Purkinje excitation.

A myocardial scar can be simulated by modifying the local conductivity and/or contractility parameters of the model. The branch block can be simulated with no excitation from the Purkinje network in the left ventricle (fig. (6)).
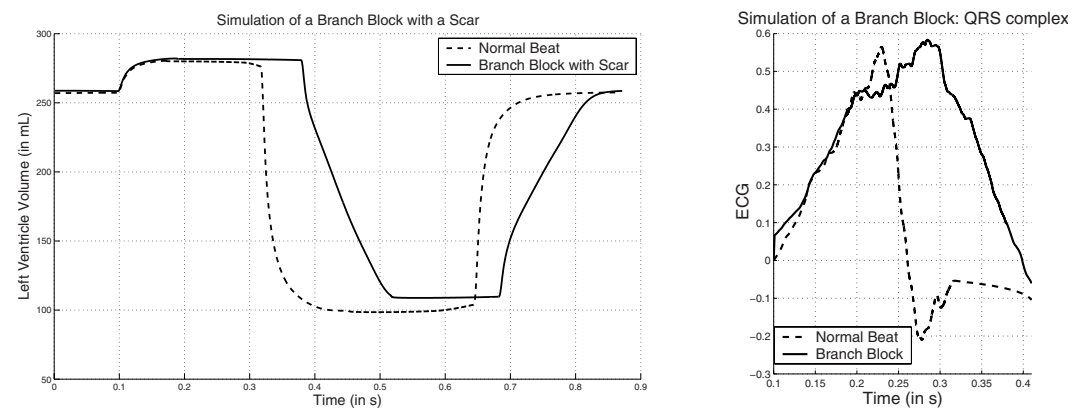

Fig. 2. Simulated left ventricle volume (left) and ECG (right) for a normal heart (dashed) and with a left branch block and a scar (solid). 


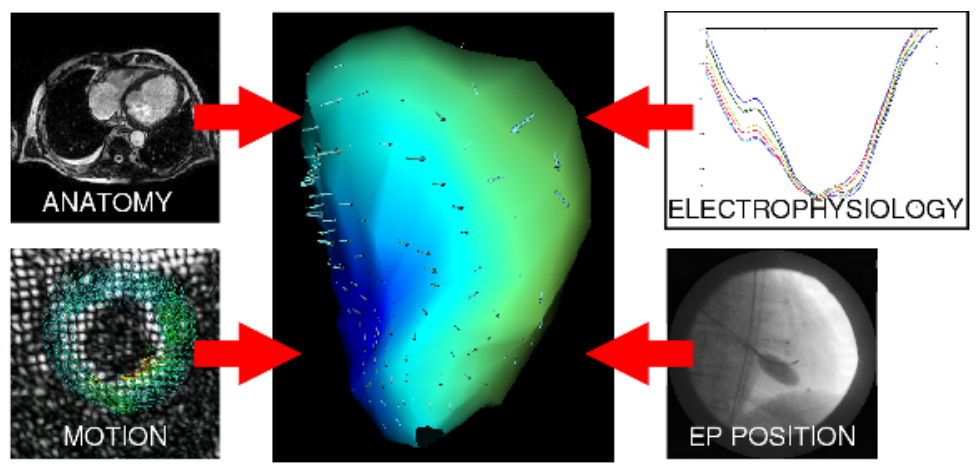

Fig. 3. Integration of the electromechanical data in the same coordinate space.
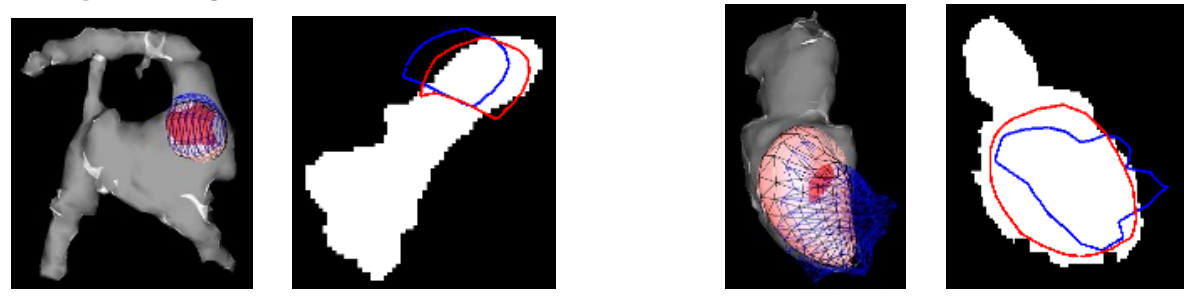

Fig. 4. Final registration between electrophysiology measures and anatomy from MRI.
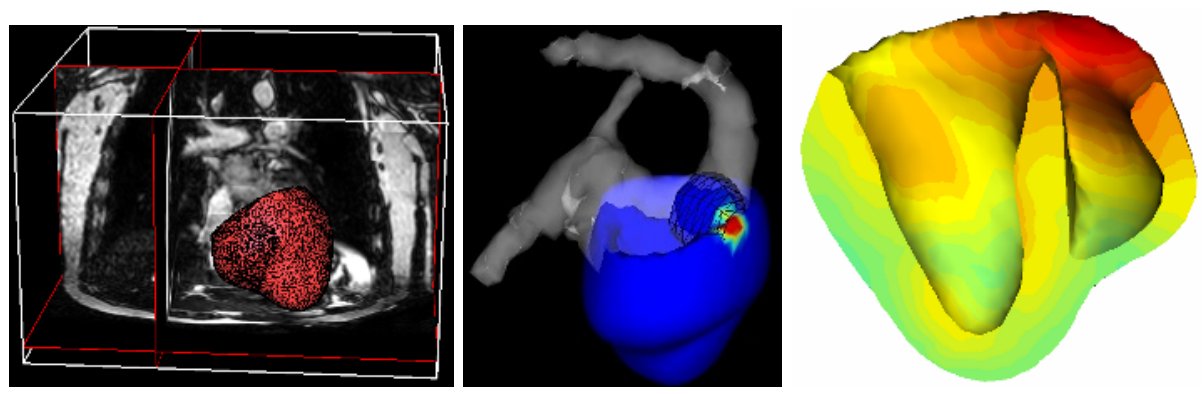

Fig. 5. Simulation of an ectopic focus from XMR measures.
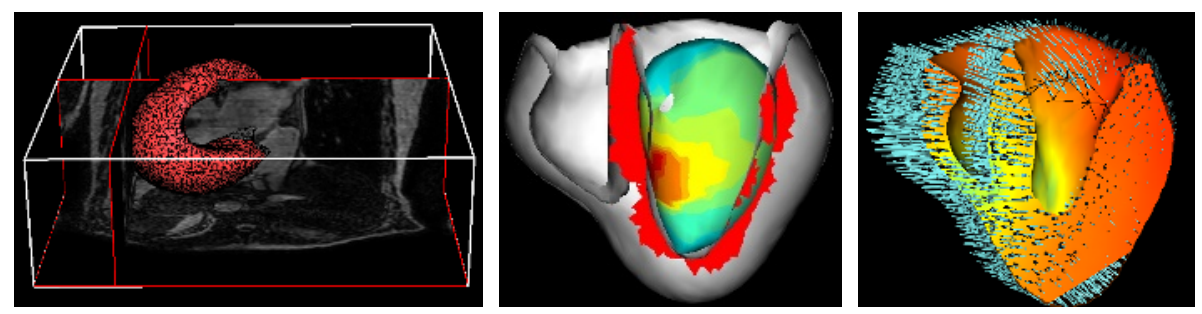

Fig. 6. Simulation of a Left Bundle Branch Block and scar from XMR measures. 
The simulated isochrones compare well with the measured ones. The simulated ECG (precordial lead, simulated with an infinite conductivity thorax [2]) shows the characteristics of a left branch block, with a larger "M" shaped QRS complex.

Moreover, the contraction is less efficient, so it needs a higher pre-load to guarantee a cardiac output high enough, which is observed in vivo.

\section{Conclusion}

In this paper we have presented a patient-specific electromechanical model of the heart. Using an XMR system, we have collected registered information about cardiac anatomy, motion and electrical activity from two patients with heart rhythm abnormalities. We have used this patient information to initialise the models, and validate the results they produce. Initial results show good agreement, and further, more quantitative validation is underway. We believe this is the first time that an electromechanical model of the heart has been clinically validated using the combination of anatomy, motion and invasive electrophysiology measurements. This validation process will lead to refinement of the model, and also enable us to identify the information required to initialise the models. Our long term aim is to use the model to devise less invasive techniques for EPS that could transform the clinical applicability and effectiveness of these procedures.

\section{Figures Legends}

Fig. 4: (left) Rigid registration of Constellation catheter surface with segmented MR. (right) Non-rigid registration of EnSite surface with segmented MR. Visualisation of initial (blue) and final (red) position and intersection with segmented MR (white).

Fig. 5: (left) Model adjusted to patient anatomy with MR. (middle) visualisation of Constellation catheter (meshed sphere), segmented anatomy (transparent), and bi-ventricular myocardium model. Colour: electrical potential when ectopic beat starts. (right) Isochrones of the simulation of the ectopic beat (red to green: 0 to $200 \mathrm{~ms})$.

Fig. 6:(left) Model adjusted to patient anatomy with MR. (middle) scar introduced in the model (red zone) from late enhancement MR and visualisation of the registered Ensite surface with measured isochrones. (right) end-systolic simulated contraction.

Acknowledgements. The authors acknowledge the UK-EPSRC (Grant JR/R41019/1), the UK Medical Imaging and Signals IRC, the UK JREI, Philips Medical Systems and the Charitable Foundation of Guy's \& St Thomas' Hospitals for funding. The authors acknowledge the contributions of Dr. E. Rosenthal, Dr. C. Bucknall, Dr. P. Lambiase, D. Elliott, ESI and the use of the MIPS software from the Epidaure project, INRIA, France. 


\section{References}

1. R. Aliev and A. Panfilov. A simple two-variable model of cardiac excitation. Chaos, Solitons 8 Fractals, 7(3):293-301, 1996.

2. O. Berenfeld and J. Jalife. Purkinje-muscle reentry as a mechanism of polymorphic ventricular arrhythmias in a 3 -dimensional model of the ventricles. Circulation Research, 82(10):1063-1077, 1998.

3. J. Bestel, F. Clément, and M. Sorine. A biomechanical model of muscle contraction. In MICCAI'01, volume 2208 of LNCS, pages 1159-1161. Springer, 2001.

4. R. Chandrashekara, R. H. Mohiaddin, and D. Rueckert. Analysis of myocardial motion in tagged MR images using non-rigid image registration. In SPIE Medical Imaging, volume 4684, pages 1168-1179, 2002.

5. M. Desbrun, M. Meyer, P. Schröder, and A. Barr. Implicit fairing of arbitrary meshes using diffusion and curvature flow. In Siggraph'99, pages 317-324. 1999.

6. P. Hunter, A. Pullan, and B. Smaill. Modeling total heart function. Annual Review of Biomedical Engineering, 5:147-177, 2003.

7. E. McVeigh, O. Faris, D. Ennis, P. Helm, and F. Evans. Measurement of ventricular wall motion, epicardial electrical mapping, and myocardial fiber angles in the same heart. In FIMH'01, number 2230 in LNCS, pages 76-82. Springer, 2001.

8. J. Montagnat and H. Delingette. Globally constrained deformable models for 3D object reconstruction. Signal Processing, 71(2):173-186, 1998.

9. K. S. Rhode, D. L. Hill, P. J. Edwards, J. Hipwell, D. Rueckert, G. Sanchez-Ortiz, S. Hegde, V. Rahunathan, and R. Razavi. Registration and tracking to integrate $\mathrm{X}$-ray and MR images in an XMR facility. IEEE Transactions on Medical Imaging, 22(11):1369-78, 2003.

10. M. Sermesant, Y. Coudière, H. Delingette, and N. Ayache. Progress towards an electro-mechanical model of the heart for cardiac image analysis. In IEEE International Symposium on Biomedical Imaging (ISBI'02), 2002.

11. M. Sermesant, O. Faris, F. Evans, E. McVeigh, Y. Coudière, H. Delingette, and N. Ayache. Preliminary validation using in vivo measures of a macroscopic electrical model of the heart. In IS4TM'03, number 2230 in LNCS. Springer, 2003.

12. N. Virag, O. Blanc, and L. Kappenberger, editors. Computer Simulation and Experimental Assessment of Cardiac Electrophysiology. Futura Publishing, 2001. 